\title{
Occipital Nerve Block for Surgery on the Posterior Scalp
}

\author{
Saad Mohammad, Ng Marilyn, Fitzpatrick Daniel, D. Kaufman, Alexander Apostol
}

SUNY Downstate Medical Center, New York, USA.

Email: saad.mohammad@downstate.edu

Received October $26^{\text {th }}, 2012$; revised November $29^{\text {th }}$, 2012; accepted December $26^{\text {th }}, 2012$

\begin{abstract}
Patients that present with scalp lesions within the distribution of cranial and spinal nerves may benefit considerably from regional nerve blocks, in this case, an occipital nerve in combination with spinal anesthesia for lower extremity anesthesia. Such blocks can be used additionally as a source of postoperative pain relief.
\end{abstract}

Keywords: Occipital Nerve; Block; Posterior Scalp; Regional Anesthesia; Skin Graft

\section{Introduction}

A 48-year-old male with a history of bipolar disorder who had previously undergone a resection of a large, exophytic fungating keloid on the posterior scalp and neck with a failed skin graft $(15 \mathrm{~cm} \times 24 \mathrm{~cm})$ was admited for a second skin graft attempt. The first attempt was performed under general anesthesia. The surgeon felt that the primary cause of initial graft failure was due to the patient's agitation perioperatively, and requested an anesthetic technique that would limit such agitation. As general anesthesia carries a risk of postoperative cognitive dysfunction, a regional anesthetic technique was implemented.

\section{Case Description}

Standard ASA monitors were applied. The patient received a spinal anesthetic with $2 \mathrm{~mL}$ of $0.5 \%$ bupivicaine (total $10 \mathrm{mg}$ ). The left thigh was chosen as the graft donor site. After confirmation via pin prick by the anesthesiologist of an adequate anesthetic level that extended from L1 caudally, the patient was placed in the semiright lateral decubitus position for skin graft procurement. Bilateral occipital nerve block scalp anesthesia was administered as an assistant held the head for optimal positioning and visualization. The scalp was prepped and draped and the occipital nerve was located along the superior nuchal line, medial to the pulsation of the occipital artery. After aspiration to rule out intravascular injection, local skin was infiltrated with $5 \mathrm{ml}$ lidocaine $2 \%$, after which aspiration was repeated to rule out intravascular injection, and $10 \mathrm{~mL}$ of bupivacaine $(0.5 \%)$ were injected on each side of the nerve. No other sedatives or medications were administered before or throughout the procedure. The patient was assessed at 5 minute intervals for pain and discomfort, neither were elicited during the procedure. The patient remained hemodynamically stable throughout the procedure. The patient reported no postoperative pain while in the PACU and was compliant with post-surgical wound instructions. After 2 hours of immediate postoperative wound observation, he was transferred to the floor for further monitoring. The patient was to be bed bound for wound care reasons. A Foley urinary catheter was placed prior to transfer as the spinal anesthetic was not yet resolved.

\section{Discussion}

Patients who present for surgery of the posterior scalp can do so for oncologic diagnoses and are often elderly with co-morbidities. General anesthesia has its own risks, which when combined with the comorbidities in such patients, adds to the problem. Compliance with postoperative instructions during immediate postoperative period is an additional concern, as in this case. Further, general anesthesia has the risk of postoperative cognitive dysfunction occurring within the elderly population. We believe that the use of appropriate regional anesthetic techniques, in this case an occipital nerve block, provides adequate anesthesia for select surgeries and avoids the risks of general anesthesia [1]. Complications of greater occipital nerve blocks are very rare. Bleeding, infection, pain, and allergic or other adverse reaction to the anesthetic or steroid are possible. The occipital nerve does not have motor function, so weakness or change in facial expression will not occur. The use of regional anesthesia can be even more beneficial in the ambulatory setting. Peripheral neurosurgical procedures already benefit from such "scalp" blocks and have been shown to be superior to local infiltration as well as attenuate hemodynamic 
responses to considerable surgical stimuli [2]. Increased use of blocks for nerves such as the supraorbital, supratrochlear, auriculotemporal, greater auricular, and greater/ lesser/third occipital nerves would be highly beneficial in providing anesthesia for lesions located within these respective nerve distributions.

\section{Conclusion}

Patients that present with scalp lesions within the distribution of cranial and spinal nerves may benefit considerably from regional nerve blocks. In this case, an occipital nerve block in combination with spinal anesthesia for lower extremity anesthesia was used. Additionally, such blocks can be a source of postoperative pain relief.

\section{REFERENCES}

[1] F. Finco, A. Matteo, et al., "Greater Occipital Nerve Block for Surgical Resection of Major Infiltrating Lesions of the Posterior Scalp," Plastic and Reconstructive Surgery, Vol. 125, No. 2, 2010, pp. 52-53. doi:10.1097/PRS.0b013e3181c72457

[2] S. Geze, et al., "The Effect of Scalp Block and Local Infiltration on the Haemodynamic and Stress Response to Skull-Pin Placement for Craniotomy,” European Journal of Anaesthesiology, Vol. 26, No. 4, 2009, pp. 298-303. doi:10.1097/EJA.0b013e32831aedb2 